\title{
NUMERICAL WAVE SCATTERING TAKING ACCOUNT OF ENERGY DISSIPATION AND MEDIA STIFFNESS AS MODELED BY THE TELEGRAPH EQUATION *
}

\author{
SEBASTIAN ACOSTA \\ Departments of Mechanical Engineering and Mathematics, Brigham Young University, Provo UT 84602 \\ email: sebastian@byu.edu \\ AND \\ PEDRO ACOSTA \\ Departments of Mathematics, and Physics and Astronomy, Brigham Young University, Provo UT 84602 \\ email: pacosta@byu.net
}

\begin{abstract}
The telegraph equation is employed to model wave fields taking into account energy dissipation and media stiffness. The timeharmonic scattered waves generated by a line source incident upon cylindrical obstacles of arbitrary cross-section are studied. Solutions are found to depend strongly on the relative values of the frequency, damping, and stiffness coefficients. These coefficients are also found to have a significant effect on the far-field pattern. The analytical solution for a circular cylinder is reviewed. An approximate finite-difference solution is also obtained for the case of a two-dimensional scatterer with an arbitrary cross-section. Details are given for both soft and hard boundary conditions. The main feature of the numerical scheme is its computational efficiency based on the coupling between boundary conforming grids and a curvilinear coordinates version of the Dirichlet-to-Neumann non-reflecting boundary condition.
\end{abstract}

Key words. Scattering, damping, stiffness, Telegraph equation, Dirichlet-to-Neumann, finite difference, curvilinear coordinates.

1. Introduction. In many applications, wave propagation experiences energy losses due to a variety of physical factors such as viscosity, heat transfer, turbulence, dispersion, electrical resistance and inhomogeneities. For example, in [9] the authors studied wave propagation through grainy materials where elastic wave attenuation is considerable. Sound decay due to viscous and thermal effects was studied by Rodarte, Temkin and others [19, 24]. Various models $[21,23,22]$ have been formulated to study high frequency wave motion in lossy media. Electromagnetic waves constitute another important example of attenuation since they are rapidly absorbed in conducting media due to the flow of free charges. In brief, attenuation plays an important role in several branches of wave propagation such as electrodynamics, elasticity, ultrasound physics, etc.

A natural way to model dissipative oscillations is to use telegraph-type equations. The telegraph equation was first derived to model the voltage and current in electrical transmission lines, such as telegraph wires. A similar dissipative model was used in [13] and [7] to study the behavior of waves. The telegraph equation has also been employed in other areas. For example, it is used as a replacement for the diffusion equation to model transport of charged particles $[10,1]$ or solar cosmic rays [8], chemical diffusion, and population dynamics [12]. It is also employed in the theory of hyperbolic heat transfer [6,5].

In this paper, the telegraph equation is used as the governing relation. It is not intended to restrict this equation to a particular wave type. Rather, it is used as a general model of scalar wave fields without specifying their actual physical nature. In the time domain, the field solution $v(\mathbf{x}, t)$ is governed by the inhomogeneous telegraph equation:

$$
\frac{1}{c^{2}} \frac{\partial^{2} v}{\partial t^{2}}+A \frac{\partial v}{\partial t}+B v-\nabla^{2} v=F
$$

By definition, $A$ and $B$ are non-negative real numbers, and they are known as the damping and stiffness coefficients, respectively. More precisely, the first order partial derivative in time term is associated with energy dissipation, and the $B v$ term corresponds to the tendency of the medium to resist deviations from a given unstressed state. This latter term is commonly encountered in modeling the vibrations of a stiff membrane that resist to be transversely deflected, regardless of the tension applied thereon. This work is directed to show the effects of damping and stiffness on the wave fields and the far-field pattern.

*Undergraduate Research Advisor: Dr. Vianey Villamizar of the Department of Mathematics, Brigham Young University, Provo, UT 84602. (vianey@math.byu.edu) 
The wave scattering of line sources from infinitely long cylindrical obstacles is studied. The obstacles are subjected to either Dirichlet (soft) or Neumann (hard) boundary conditions. In particular, focus is placed on time-harmonic waves subjected to various degrees of energy dissipation and media stiffness. Time-harmonic solutions of the form $v(\mathbf{x}, t)=u(\mathbf{x}) e^{-i \omega t}$ are sought. They are induced by time-harmonic line sources of the form $F=f_{0}(\mathbf{x}) e^{-i \omega t}$ which leads to the reduced telegraph equation:

$$
\nabla^{2} u+k^{2} u=-f_{0}
$$

where $k^{2}=\omega^{2} / c^{2}+A \omega i-B$. It is noticed that the problem reduces to the Helmholtz equation with a complex wave number.

The exact solution to the scattering problem governed by equation (1.2) in the presence of a circular cylinder (with soft and hard boundary conditions) is reviewed, and a numerical method is applied for a cylinder of arbitrary cross-section. The numerical method consists of an implicit finite-difference scheme supported by boundary-fitted elliptic grids.

Our numerical method requires that the unbounded physical domain is truncated. In fact, the radiation condition at infinity is replaced by a Dirichlet-to-Neumann (DtN) absorbing boundary condition over a circle enclosing the obstacle (as shown in Figure 2.1). For completeness, the derivation of the DtN boundary condition, first obtained by Keller and Givoli in [14], is presented and incorporated into our numerical method. To the best of our knowledge, this is the first work in which the DtN condition is expressed in terms of generalized curvilinear coordinates and coupled with elliptic grids. The major goal of the numerical part of this work is to obtain accurate solutions for arbitrary scatterers by means of a computationally feasible method. As it will be shown later, this goal is met since the computational region is considerably reduced by using the DtN condition and by combining it with generalized curvilinear boundary conforming coordinates.

The outline of the paper is as follows. In Section 2, an expression for the incident field is found to be the twodimensional Green's function for Helmholtz equation with a complex wave number. In Section 3 , the scattering boundary value problem modeled by the reduced telegraph equation is considered. For clarity, some details on the derivation of its analytical solution, based on eigenfunction expansions, for the circular scatterer are given. In Section 4 , the derivation of the DtN non-reflecting boundary condition for the reduced telegraph equation is reconsidered in preparation for the numerical treatment of our boundary value problem. One of the goals of this work is to study the scattering of waves from obstacles of arbitrary shape when the reduced telegraph equation is used as a governing equation. This is the subject of Section 5. To achieve this goal, the entire governing boundary value problem (BVP) is expressed in curvilinear coordinates that conform to the bounding curve of the scatterers. Section 6 deals with the numerical solution for complexly shaped scatterers. The grid generation process is described, and the finite-difference method in curvilinear coordinates used to solve the problem is devised. In Section 7, the formula for the far-field pattern is discussed. In Section 8, the numerical solution is compared with the exact solution for the circular cylinder as a validation procedure. Then, the method is applied to a complexly shaped scatterer, and the effects of damping and stiffness are analyzed. Finally, Section 9 contains our concluding remarks.

2. The Incident Field. Consider the wave scattering of a line source from an infinitely long cylinder of arbitrary cross-section. The axes of the line source and of the cylinder are taken parallel to the $z$-axis. This symmetry leads to a two-dimensional problem. A global Cartesian system of coordinates $(x, y)$ is defined with its origin on the axis of the cylindrical obstacle. An arbitrary point in this system has a vector position denoted by $\mathbf{x}=(x, y)$ and its corresponding polar coordinates $(r, \theta)$ such that $x=r \cos \theta$ and $y=r \sin \theta$. A second Cartesian coordinate system $(\tilde{x}, \tilde{y})$ is defined with its origin at the source (see Figure 2.1), and associated polar coordinates $(\tilde{r}, \tilde{\theta})$ such that $\tilde{x}=\tilde{r} \cos \tilde{\theta}$ and $\tilde{y}=r \sin \tilde{\theta}$. The location of the origin of the first polar coordinate system with respect to the second one is $(L, \phi)$.

The time-harmonic solution $u(r, \theta)$ is induced by the two-dimensional source $f_{0}=-\Lambda \delta(\tilde{x}) \delta(\tilde{y})$ acting in the plane. This source can be expressed in polar coordinates as $f_{0}=-\Lambda \delta(\tilde{r}) /(2 \pi \tilde{r})$, where $\delta$ is the one-dimensional Dirac delta function and $\Lambda$ is the strength of the source. For a complete discussion on delta functions for multidimensional spaces, the reader is referred to Chapter 9 of book titled Green's Functions by Roach [18]. Let the total field be decomposed into an incident $u_{i n c}(\tilde{r})$ and a scattered field $u_{s c}(r, \theta)$, such that

$$
u(r, \theta)=u_{i n c}(\tilde{r})+u_{s c}(r, \theta)
$$




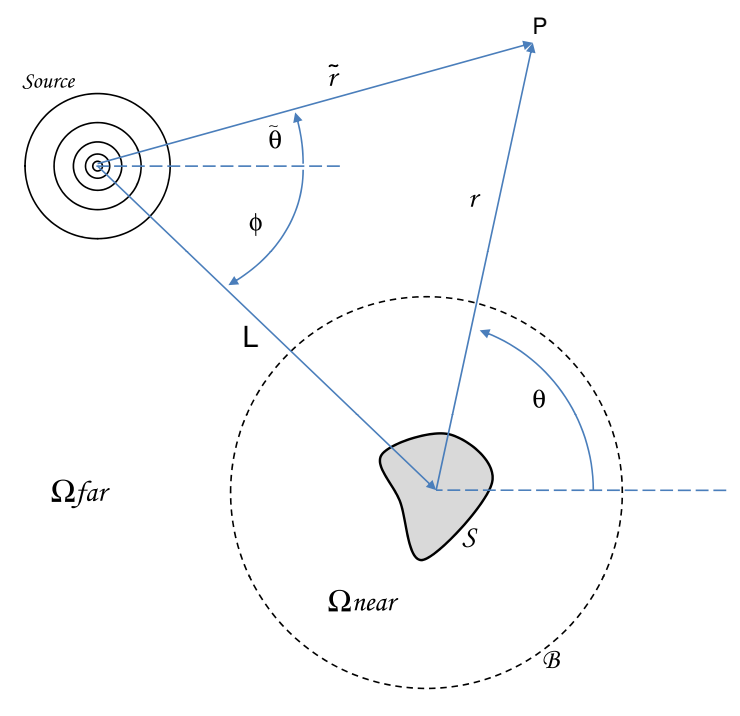

FIG. 2.1. Sketch of the physical domain, the source, and the coordinate systems.

The incident field satisfies the following equation:

$$
\frac{1}{\tilde{r}} \frac{d}{d \tilde{r}}\left(\tilde{r} \frac{d}{d \tilde{r}} u_{i n c}\right)+k^{2} u_{i n c}=\Lambda \frac{\delta(\tilde{r})}{2 \pi \tilde{r}} \quad \text { in } \mathbb{R}^{2}
$$

It is noticed that there is no $\tilde{\theta}$-dependence in equation (2.2) due to the radial symmetry of the line source. Since the original line source reduces to a single source in two dimensions, then the incident field happens to be the twodimensional fundamental solution (amplified by $\Lambda$ ) of the reduced telegraph equation, that is

$$
u_{i n c}(\tilde{r})=\frac{-i \Lambda}{4} H_{0}^{(1)}(k \tilde{r}) .
$$

This is the same infinite plane Green's function for the Helmholtz equation with the only difference that the wave number $k$ is a complex number in general. This expression for the incident field is used not only in the analytical Section 3 of the present article, but also in the subsequent numerical treatment. From this point on, we will work on obtaining the scattered field $u_{s c}$, keeping in mind that the incident field $u_{i n c}$ plays an important role by determining the scattered field $u_{s c}$ at the boundary of the obstacle. Furthermore, in order to obtain the total field $u$, it is necessary to superimpose both of its components as in Equation (2.1).

3. Analytical Solution for a Scatterer with Circular Cross-section. Consider the wave scattering from a circular cylinder of radius $a$. The scattered field $u_{s c}$ satisfies the following boundary value problem in an infinite domain $\Omega$ bounded internally by a soft cylindrical surface $\mathcal{S}$ :

$$
\begin{aligned}
& \nabla^{2} u_{s c}+k^{2} u_{s c}=0 \text { in } \Omega, \\
& u_{s c}=-u_{i n c} \text { on } \mathcal{S} \text {, } \\
& \sqrt{r}\left(\frac{\partial u_{s c}}{\partial r}-i k u_{s c}\right) \rightarrow 0 \quad \text { as } \quad r \rightarrow \infty,
\end{aligned}
$$

where Equation (3.3) is the Sommerfeld radiation condition. For a hard cylindrical surface, Equation (3.2) is replaced by

$$
\frac{\partial u_{s c}}{\partial r}=-\frac{\partial u_{i n c}}{\partial r} \quad \text { on } \mathcal{S}
$$


At this point, it is appropriate to write the incident field in terms of the first polar coordinate system. In other words, in terms of $r$ and $\theta$. This is an essential step in order to find the scattered field. To do so, Graf's addition theorems are applied. Excellent discussions of these theorems are found in Chapter 2 of [17] and Chapter 10 of [29]. For the Hankel functions, they state that

$$
H_{m}^{(1)}(k \tilde{r}) e^{i m \tilde{\theta}}=\sum_{n=-\infty}^{\infty} H_{m-n}^{(1)}(k L) J_{n}(k r) e^{i n \theta} e^{i(m-n) \phi} \quad \text { for } \quad m=0,1,2, \ldots
$$

In particular, it is necessary to find $H_{0}^{(1)}$ by setting $m=0$, and use the identity $H_{-n}^{(1)}(k L)=(-1)^{n} H_{n}^{(1)}(k L)$, to expand the incident field in terms of the first polar coordinate system as follows:

$$
u_{i n c}(r, \theta)=\frac{-i \Lambda}{4} \sum_{n=-\infty}^{\infty}(-1)^{n} H_{n}^{(1)}(k L) J_{n}(k r) e^{i n(\theta-\phi)} .
$$

We are now in a position to find the scattered field. In terms of the eigenfunctions of the corresponding homogeneous BVP, solutions to Equation (3.1) are given by

$$
u_{s c}(r, \theta)=\frac{i \Lambda}{4} \sum_{n=-\infty}^{\infty}(-1)^{n} C_{n} H_{n}^{(1)}(k L) H_{n}^{(1)}(k r) e^{i n(\theta-\phi)} .
$$

Since the Hankel functions automatically satisfy the Sommerfeld radiation condition, it only remains to satisfy the condition at the obstacle's boundary, either (3.2) or (3.4). Using the known coefficients of the expansion of the incident wave (3.6), the unknown coefficients of the infinite series (3.7) for the scattered wave are determined. In fact, for the soft obstacle boundary the coefficients are

$$
C_{n}=\frac{J_{n}(k a)}{H_{n}^{(1)}(k a)}
$$

whereas for the hard one, Equation (3.4) yields

$$
C_{n}=\frac{J_{n}^{\prime}(k a)}{H_{n}^{(1)}(k a)} .
$$

For computational reasons, the symmetry about the line passing through the source and the center of the cylinder is exploited. Thus, the scattered field may be written as

$$
u_{s c}(r, \theta)=\frac{i \Lambda}{4} \sum_{n=0}^{\infty} \epsilon_{n}(-1)^{n} C_{n} H_{n}^{(1)}(k L) H_{n}^{(1)}(k r) \cos n(\theta-\phi)
$$

where $\epsilon_{n}$ is the Neumann factor, ie. $\epsilon_{0}=1$ and $\epsilon_{n}=2$ for $n \geq 1$.

This exact solution will help us to find the far-field pattern in Section 7, and it will be used to validate our numerical method in Section 8. Moreover, from the analytical solution, much may be learned about the behavior of the fields for different values of $A$ and $B$. Notice that the damping and stiffness coefficients are embedded into the effective wave number $k$. This number will induce very different wave behavior depending on whether $k$ is a purely imaginary number, purely real, or neither. These differences are made evident by analyzing the asymptotic expansions of the Hankel functions (see Chapter 9 of the Handbook of Mathematical Functions [2] or Chapter 7 of [29]) that appear in the incident and scattered fields. Indeed, for large arguments $(|z| \rightarrow \infty)$ and any integer order $n$,

$$
H_{n}^{(1)}(z) \rightarrow(-i)^{n} \frac{(1-i)}{\sqrt{\pi z}} e^{i z}
$$

where $z=k r$. Notice that there is a decaying factor that is inversely proportional to $\sqrt{r}$. This is due to the fact that the radiating waves are spreading over the two-dimensional space as they propagate. However, there is an additional exponentially decaying term if $z$ has a positive imaginary part.

Although the wave fields are continuously oscillating in the time domain with an angular frequency $w$, it is the radial profile of oscillation in the frequency domain what we are after. These spatial profiles may be classified in the following three cases or regimes: 
- Case 1: The effective wave number $k$ is purely imaginary or identically zero. This occurs when there is no damping at all and $B \geq w^{2} / c^{2}$. In this case, a non-oscillatory radial profile results. In fact, the Hankel functions may be replaced by the modified Bessel functions of second kind (or MacDonald's functions) which are well-known to decay without oscillations. Notice that only the relative difference between $B$ and $w^{2} / c^{2}$ is relevant but not their actual values. The greater this difference, the more rapidly waves will decay over distance. Also, it is worth noting that $B=w^{2} / c^{2}$ is a special case in which the governing equation reduces to Laplace equation.

- Case 2: The effective wave number is real. Again, this occurs when there is no damping at all, but $w^{2} / c^{2}>$ $B$. In this case, the radial profile consists of oscillations. Moreover, the greater the difference between $w^{2} / c^{2}$ and $B$ is, the more spatial oscillations will fit in a given unit of radial distance.

- Case 3: The effective wave number has non-zero, real and imaginary parts. This case occurs whenever $A>0$ (for $A<0$ the solution is non-physical). The resulting field combines the behavior of the previous cases. There will be spatial oscillations as well as damping effects in the amplitude.

The real part of the incident field (2.3) for these three cases is illustrated in Figure 3.1. Here, the following parameters, $c=1, \Lambda=1$ and $w=2 \pi$, are taken for all cases.

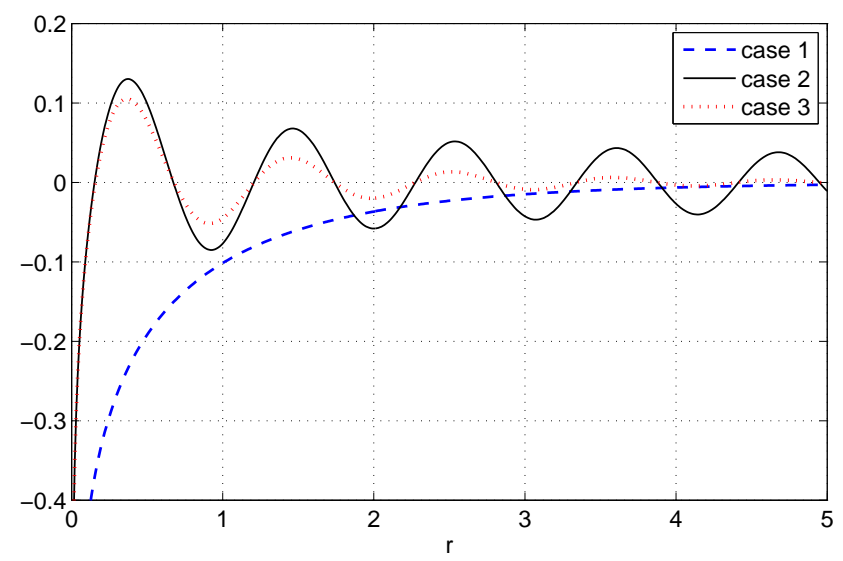

FIG. 3.1. Illustration of the radial wave profile of the incident field (real part) for the three different cases. For Case 1: $A=0$ and $B=40$, for Case 2: $A=0$ and $B=5$, and for Case 3: $A=1$ and $B=5$.

4. Dirichlet-to-Neumann Boundary Condition. In this section, a review of the derivation of the Dirichlet-toNeumann (DtN) absorbing boundary condition for the Helmholtz equation is presented. It will be used in Section 6 for the numerical treatment of the BVP (3.1)-(3.4).

The DtN condition was first derived in [14]. It is a non-reflecting boundary condition especially developed to obtain numerical solutions to BVP for Laplace and Helmholtz equations in unbounded domains. It enables the efficient replacement of the physical unbounded region by a bounded domain. This condition permits waves to leave the truncated region without any spurious or non-physical reflections.

The first step in the derivation of the DtN condition is to divide the physical domain $\Omega$ in two regions: A bounded sub-domain $\Omega_{\text {near }}$ that contains the obstacle, and the remaining unbounded exterior region $\Omega_{f a r}$, as shown in Figure 2.1. The interfacial boundary between these regions is denoted by $\mathcal{B}$. In general, $\mathcal{B}$ should be described by a curve that coincides with a coordinate line of a separable system of coordinates. In this work, $\mathcal{B}$ is chosen to be a circle of radius $R$ centered at the origin.

The second step is to solve the Dirichlet problem in the unbounded region $\Omega_{f a r}$ by assuming the field $u_{s c}$ to be known on $\mathcal{B}$ and the Sommerfeld radiation condition at infinity. The well-known solution for this BVP can be expressed as the following series of eigenfunctions:

$$
u_{s c}(r, \theta)=\frac{1}{2 \pi} \sum_{n=0}^{\infty} \epsilon_{n} \frac{H_{n}^{(1)}(k r)}{H_{n}^{(1)}(k R)} \int_{0}^{2 \pi} u_{s c}(R, \bar{\theta}) \cos n(\theta-\bar{\theta}) d \bar{\theta}
$$


where $r \geq R$. If the wave number $k$ happens to be identically zero, then the governing equation reduces to Laplace equation which belongs to Case 1. As it was noted in [14], Equation (4.1) may be replaced by its limiting form as the argument of the Hankel functions tends to zero. In fact, as shown in page 360, Chapter 9 of reference [2], the limiting behavior for small arguments $(z \rightarrow 0)$ is the following:

$$
\begin{aligned}
& H_{0}^{(1)}(z) \rightarrow 1+\frac{2 i}{\pi} \ln z \quad \text { and } \\
& H_{n}^{(1)}(z) \rightarrow \frac{-i}{\pi}(n-1) !(z / 2)^{-n} \quad \text { for } n>0,
\end{aligned}
$$

which leads to

$$
\frac{H_{n}^{(1)}(k r)}{H_{n}^{(1)}(k R)} \rightarrow\left(\frac{R}{r}\right)^{n} \quad \text { as } \quad k \rightarrow 0 \quad \text { for } n \geq 0,
$$

yielding the exact solution for Laplace equation in the unbounded domain $\Omega_{f a r}$ for a given Dirichlet-type data at $r=R$,

$$
u_{s c}(r, \theta)=\frac{1}{2 \pi} \sum_{n=0}^{\infty} \epsilon_{n}\left(\frac{R}{r}\right)^{n} \int_{0}^{2 \pi} u_{s c}(R, \bar{\theta}) \cos n(\theta-\bar{\theta}) d \bar{\theta} .
$$

The analytical solution (4.1) in $\Omega_{f a r}$ renders an exact relation between the unknown function $u_{s c}$ and its normal derivative on $\mathcal{B}$. Therefore, the final step is to differentiate Equation (4.1) with respect to $r$ and set $r=R$, which results in the DtN boundary condition for Helmholtz equation:

$$
\frac{\partial u_{s c}}{\partial r}(R, \theta)=\frac{k}{2 \pi} \sum_{n=0}^{\infty} \epsilon_{n} \frac{H_{n}^{(1) \prime}(k R)}{H_{n}^{(1)}(k R)} \int_{0}^{2 \pi} u_{s c}(R, \bar{\theta}) \cos n(\theta-\bar{\theta}) d \bar{\theta} .
$$

For the limiting case when $k \rightarrow 0$, it is observed that

$$
k \frac{H_{n}^{(1) \prime}(k R)}{H_{n}^{(1)}(k R)} \rightarrow-\frac{n}{R},
$$

and the corresponding DtN boundary condition for Laplace equation

$$
\frac{\partial u_{s c}}{\partial r}(R, \theta)=\frac{-1}{2 \pi} \sum_{n=0}^{\infty} \epsilon_{n} \frac{n}{R} \int_{0}^{2 \pi} u_{s c}(R, \bar{\theta}) \cos n(\theta-\bar{\theta}) d \bar{\theta}
$$

is obtained. Although no problem is treated in this paper for which $k$ is exactly zero, Equation (4.7) is provided for completeness.

Boundary condition (4.5), prescribed at the interfacial circle $\mathcal{B}$, completes the formulation of the BVP in $\Omega_{\text {near }}$. This BVP will be solved numerically. Due to the arbitrariness of the obstacle's bounding curve, a curvilinear coordinate system is employed in the bounded sub-domain. This requires to express the DtN condition (4.5) in terms of generalized curvilinear coordinates, which is done in Section 5.

5. Boundary Conforming Coordinates. One of the goals of this work is to develop efficient and reliable numerical methods to obtain approximations for the solution of the scattering problem modeled by (3.1)-(3.4). An important part of the technique is to create an appropriate grid for the region $\Omega_{\text {near }}$ that contains a complexly shaped obstacle. Our approach is based on creating a grid that fits the arbitrary inner boundary $\mathcal{S}$ and the circular interfacial boundary $\mathcal{B}$.

The grid generation method is due to Winslow [30]. Once the grid is constructed, it will be used in the finitedifference treatment of our problem. The Winslow coordinates may be regarded as a transformation $T$ from a computational domain $\mathcal{C}$ with rectangular coordinates $(\xi, \eta)$ to the truncated physical domain $\Omega_{\text {near }}$ with coordinates $(x(\xi, \eta), y(\xi, \eta))$. The cylinder's bounding curve is denoted by $\mathcal{S}$, and the artificial boundary by $\mathcal{B}$, as seen in Figure 2.1. The computational variables are such that $1 \leq \xi \leq N_{1}$ and $1 \leq \eta \leq N_{2}$. An illustration of this transformation 
with its domain and range is displayed in Figure 5.1. The transformation $T$ is implicitly defined as the solution of the following Dirichlet boundary value problem governed by the two quasi-linear elliptic equations:

$$
\begin{aligned}
& \alpha x_{\xi \xi}-2 \beta x_{\xi \eta}+\gamma x_{\eta \eta}=0 \\
& \alpha y_{\xi \xi}-2 \beta y_{\xi \eta}+\gamma y_{\eta \eta}=0
\end{aligned}
$$

where the Dirichlet-type boundary data is given by specifying the coordinates $(x, y)$ at the boundaries of $\Omega_{n e a r}$ such that the parametric curves $(x(\xi, 1), y(\xi, 1))$ and $\left(x\left(\xi, N_{2}\right), y\left(\xi, N_{2}\right)\right)$ coincide with the obstacles's boundary $\mathcal{S}$ and the artificial boundary $\mathcal{B}$, respectively. The symbols $\alpha, \beta$ and $\gamma$ represent the scale metric factors of the transformation $T$. They are defined as $\alpha=x_{\eta}^{2}+y_{\eta}^{2}, \beta=x_{\xi} x_{\eta}+y_{\xi} y_{\eta}$, and $\gamma=x_{\xi}^{2}+y_{\xi}^{2}$. The jacobian $J$ of this transformation plays an important role in the formulation of the scattering problem in curvilinear coordinates. It is defined as $J=x_{\xi} y_{\eta}-x_{\eta} y_{\xi}$.

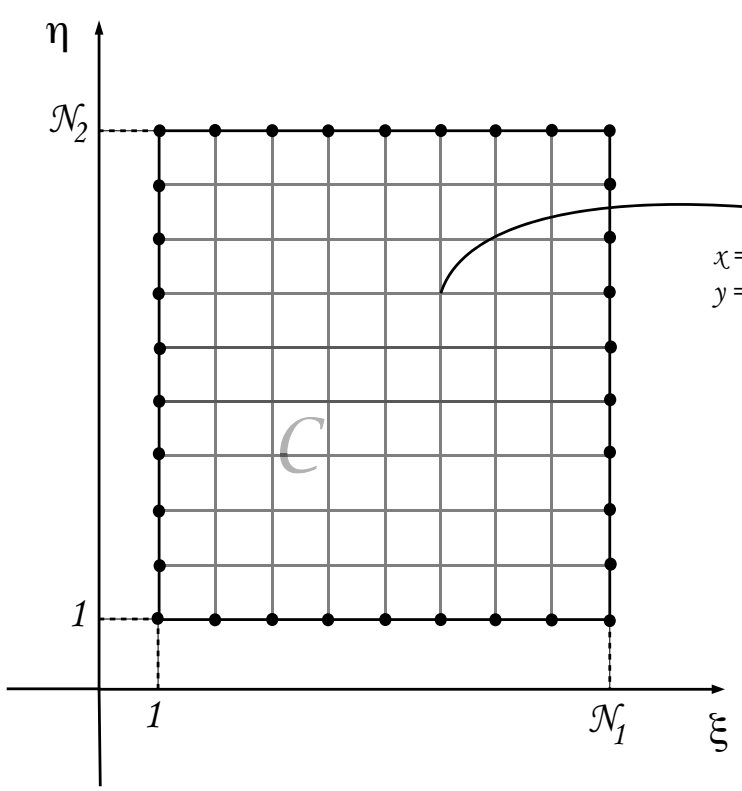

Computational Domain

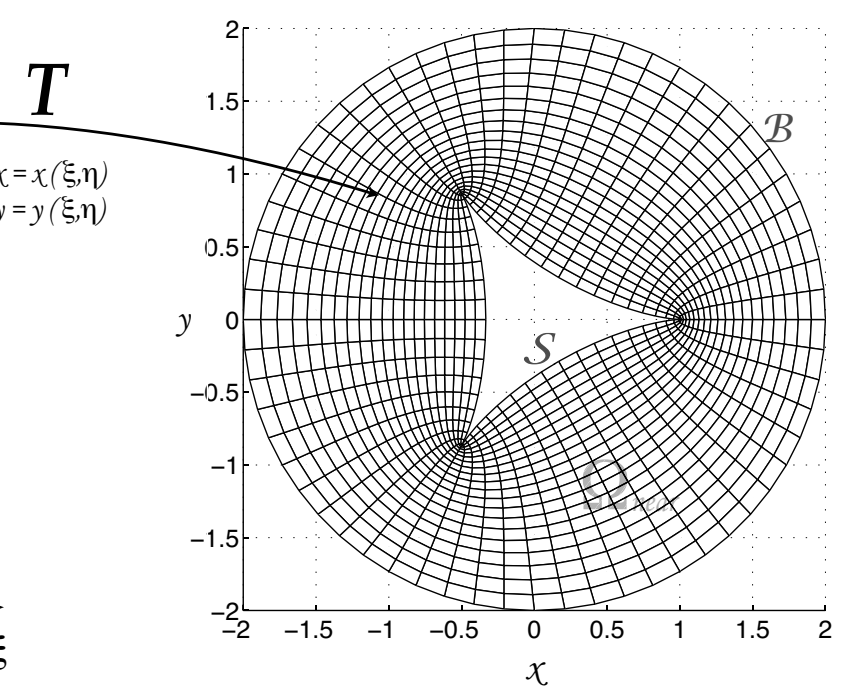

Physical Domain

FIG. 5.1. Illustration of the transformation $T$ that is used in the generation of the boundary conforming coordinates.

At this point, the entire scattering boundary value problem in $\Omega_{\text {near }}$ may be written in terms of generalized curvilinear coordinates $(\xi, \eta)$. In particular, explicit expressions are sought for the Laplacian and the gradient operators acting on the scattered field. A complete derivation is provided in Section 2.3 of the second Chapter of [15]. The final expressions for the components of the gradient are:

$$
\begin{aligned}
\left(u_{s c}\right)_{x} & =\frac{1}{J}\left(\left(u_{s c}\right)_{\xi} y_{\eta}-\left(u_{s c}\right)_{\eta} y_{\xi}\right), \\
\left(u_{s c}\right)_{y} & =\frac{1}{J}\left(\left(u_{s c}\right)_{\eta} x_{\xi}-\left(u_{s c}\right)_{\xi} x_{\eta}\right),
\end{aligned}
$$

and the Laplacian becomes:

$$
\begin{aligned}
& \nabla^{2} u_{s c}=\left(u_{s c}\right)_{x x}+\left(u_{s c}\right)_{y y}=\frac{1}{J^{2}}\left(\alpha\left(u_{s c}\right)_{\xi \xi}-2 \beta\left(u_{s c}\right)_{\xi \eta}+\gamma\left(u_{s c}\right)_{\eta \eta}\right) \\
& +\frac{1}{J^{3}}\left(\alpha x_{\xi \xi}-2 \beta x_{\xi \eta}+\gamma x_{\eta \eta}\right)\left(y_{\xi}\left(u_{s c}\right)_{\eta}-y_{\eta}\left(u_{s c}\right)_{\xi}\right) \\
& +\frac{1}{J^{3}}\left(\alpha y_{\xi \xi}-2 \beta y_{\xi \eta}+\gamma y_{\eta \eta}\right)\left(x_{\eta}\left(u_{s c}\right)_{\xi}-x_{\xi}\left(u_{s c}\right)_{\eta}\right) .
\end{aligned}
$$


The above expressions are completely general and valid for any system of coordinates. However, the Laplacian may be simplified considerably by using the Winslow coordinates and substituting Equations (5.1)-(5.2) into it. The result is the following:

$$
\nabla^{2} u_{s c}=\frac{1}{J^{2}}\left(\alpha\left(u_{s c}\right)_{\xi \xi}-2 \beta\left(u_{s c}\right)_{\xi \eta}+\gamma\left(u_{s c}\right)_{\eta \eta}\right)
$$

The Winslow coordinates provide a means to construct boundary-fitted curvilinear coordinates and also simplify the cumbersome expression for the Laplacian operator. Another major advantage of this elliptic approach is that the interior coordinates are smooth, even for non-smooth boundary data. Thus, the Helmholtz equation and the boundary condition at the obstacle's surface may be written in terms of $(\xi, \eta)$ as follows:

$$
\begin{gathered}
\frac{1}{J^{2}}\left(\alpha\left(u_{s c}\right)_{\xi \xi}-2 \beta\left(u_{s c}\right)_{\xi \eta}+\gamma\left(u_{s c}\right)_{\eta \eta}\right)+k^{2} u_{s c}=0 \\
u_{s c}(\xi, 1)=-u_{i n c}(\xi, 1) \quad \text { on soft } \mathcal{S}, \text { or } \\
\frac{\partial u_{s c}}{\partial n}(\xi, 1)=-\frac{\partial u_{i n c}}{\partial n}(\xi, 1) \quad \text { on hard } \mathcal{S}
\end{gathered}
$$

Furthermore, the incident field may be introduced since it is already known. For the soft obstacle, the Dirichlet boundary condition is obtained in terms of $(\xi, \eta)$ by substituting the incident field $u_{i n c}$ from Equation (2.3) into Equation (5.7). The following is the effective condition on the soft physical boundary $\mathcal{S}$ :

$$
u_{s c}=\frac{i \Lambda}{4} H_{0}^{(1)}(k \tilde{r}) \quad \text { at } \eta=1,
$$

where $\tilde{r}=\sqrt{\left(x-x_{s}\right)^{2}+\left(y-y_{s}\right)^{2}}$ and $\left(x_{s}, y_{s}\right)$ is the location of the source in the global Cartesian system of coordinates.

For the hard obstacle, the Neumann boundary condition is more involved. In Equation (5.8), there are partial derivatives of $u_{s c}$ and $u_{i n c}$ in the normal direction to $\mathcal{S}$. They may be expressed in terms of $(\xi, \eta)$ by using the gradients (5.3)-(5.4) as follows:

$$
\frac{\partial u_{s c}}{\partial n}=\hat{\mathbf{n}} \cdot \nabla u_{s c}=\frac{1}{\sqrt{\gamma}}\left(\begin{array}{c}
-y_{\xi} \\
x_{\xi}
\end{array}\right) \cdot \frac{1}{J}\left(\begin{array}{c}
\left(u_{s c}\right)_{\xi} y_{\eta}-\left(u_{s c}\right)_{\eta} y_{\xi} \\
\left(u_{s c}\right)_{\eta} x_{\xi}-\left(u_{s c}\right)_{\xi} x_{\eta}
\end{array}\right),
$$

where the unit normal vector $\hat{\mathbf{n}}$ and the gradient of $u_{s c}$ have been written in terms of the generalized curvilinear coordinates $(\xi, \eta)$. By substituting $u_{i n c}$ from Equation (2.3) into Equation (5.8), the partial derivative of the incident field becomes:

$$
\frac{\partial u_{i n c}}{\partial n}=\hat{\mathbf{n}} \cdot \nabla u_{i n c}=\frac{1}{\sqrt{\gamma}}\left(\begin{array}{c}
-y_{\xi} \\
x_{\xi}
\end{array}\right) \cdot \frac{-i \Lambda k H_{0}^{(1) \prime}(k \tilde{r})}{4 \tilde{r}}\left(\begin{array}{c}
x-x_{s} \\
y-y_{s}
\end{array}\right)
$$

Combining these results, condition (5.8) at the hard physical boundary $\mathcal{S}$ becomes:

$$
\gamma\left(u_{s c}\right)_{\eta}-\beta\left(u_{s c}\right)_{\xi}=\frac{i \Lambda k J}{4 \tilde{r}}\left(\left(y-y_{s}\right) x_{\xi}-\left(x-x_{s}\right) y_{\xi}\right) H_{0}^{(1) \prime}(k \tilde{r}) \quad \text { at } \eta=1
$$

Finally, it only remains to express the DtN non-reflecting boundary condition (4.5) on $\mathcal{B}$ in terms of generalized curvilinear coordinates. Now we have a partial derivative in the direction normal to the artificial boundary $\mathcal{B}$. Again, this is written as the dot product between the unit normal vector $\hat{\mathbf{n}}$ and the gradient of $u_{s c}$. Since $\mathcal{B}$ is a circle of radius $R$ centered at the origin, then it is convenient to write the unit vector as follows: $\hat{\mathbf{n}}=(1 / R)\left(x\left(\xi, N_{2}\right), y\left(\xi, N_{2}\right)\right)$. Consequently, the partial derivative of $u_{s c}$ in the normal direction to $\mathcal{B}$ becomes:

$$
\frac{\partial u_{s c}}{\partial n}=\hat{\mathbf{n}} \cdot \nabla u_{s c}=\frac{1}{R}\left(\begin{array}{c}
x \\
y
\end{array}\right) \cdot \frac{1}{J}\left(\begin{array}{c}
\left(u_{s c}\right)_{\xi} y_{\eta}-\left(u_{s c}\right)_{\eta} y_{\xi} \\
\left(u_{s c}\right)_{\eta} x_{\xi}-\left(u_{s c}\right)_{\xi} x_{\eta}
\end{array}\right) .
$$


Substituting this last result into Equation (4.5), the DtN non-reflecting boundary condition on $\mathcal{B}$ adopts the following form:

$$
\begin{aligned}
& \frac{1}{R J}\left(\left(x y_{\eta}-y x_{\eta}\right)\left(u_{s c}\right)_{\xi}+\left(y x_{\xi}-x y_{\xi}\right)\left(u_{s c}\right)_{\eta}\right) \\
& =\frac{k}{2 \pi} \sum_{n=0}^{\infty} \epsilon_{n} \frac{H_{n}^{(1)}{ }^{\prime}(k R)}{H_{n}^{(1)}(k R)} \int_{1}^{N_{1}} u_{s c}\left(\bar{\xi}, N_{2}\right) \cos n(\theta(\xi)-\theta(\bar{\xi})) \theta^{\prime}(\bar{\xi}) d \bar{\xi} \quad \text { at } \eta=N_{2} .
\end{aligned}
$$

Notice that on the circle $\mathcal{B}$, the polar angle $\theta$ can be written in terms of the independent variable $\xi$ such that $\theta=\theta(\xi)$. The actual dependence on $\xi$ is explicitly obtained once the parametrization $\left(x\left(\xi, N_{2}\right), y\left(\xi, N_{2}\right)\right)$ is established.

The Equations (5.6), (5.9), (5.10) and (5.11) represent the scattering BVP in terms of Winslow curvilinear coordinates. Details of the grid generation process and the numerical solution of this scattering problem are offered in the next section.

6. Numerical Solution for a Scatterer with Arbitrary Cross-section. This section is concerned with the construction of the numerical method that is employed to solve the scattering problem for obstacles of arbitrary crosssection. These obstacles may include geometric singularities such as cusps and corners. Due to this geometric complexity, analytical solutions are not generally available.

An implicit finite-difference scheme is chosen to approximate the solution of BVP (5.6)-(5.11) in Winslow curvilinear coordinates. The numerical technique is intended to approximate the scattered field in the vicinity of a complexly shaped obstacle and also to approximate the corresponding far-field pattern. By varying the values of the coefficients $A$ and $B$, the effects of damping and stiffness are analyzed. In particular, all the cases described in Section 3 are considered. The grid generation constitutes the first step of the numerical method.

6.1. Grid Generation Process. Equations (5.1)-(5.2) are numerically solved using a second order finite-difference scheme. For convenience, the discretization procedure is based on a uniform partition of the independent variables $\xi$ and $\eta$ such that $\Delta \xi=\Delta \eta=1$ where $\Delta$ denotes the cell size in the computational domain $\mathcal{C}$. The discrete values of $\xi$ and $\eta$ are represented by $\xi_{i}=i \Delta \xi=i$ and $\eta_{j}=j \Delta \eta=j$, for $i=1,2, \ldots, N_{1}$ and $j=1,2, \ldots, N_{2}$, respectively. Also, the discrete values of the dependent variables $x\left(\xi_{i}, \eta_{j}\right)$ and $y\left(\xi_{i}, \eta_{j}\right)$ are represented by $x_{i, j}$ and $y_{i, j}$, respectively. An analogous notation is used for the discrete values of $\alpha, \beta, \gamma$, and $J$. The grid size is denoted by $N_{1} \times N_{2}$. A refinement of the discretization is then obtained by increasing $N_{1}$ and $N_{2}$ as desired.

When the partial derivative terms in Equations (5.1)-(5.2) are approximated by centered finite-difference formulas, an algebraic equation is obtained for each discrete value of $x$ and $y$. As a result, twice as many equations as interior points in the Winslow grid are obtained. For the $x$-component of the interior grid points $\left(x_{i, j}, y_{i, j}\right)$, the equation is written in point successive over relaxation (SOR) iterative form as follows:

$$
\begin{aligned}
& x_{i, j}^{l}=\frac{1}{2(\alpha+\gamma)_{i, j}}\left[\alpha_{i, j}\left(x_{i+1, j}^{l-1}+x_{i-1, j}^{l}\right)+\gamma_{i, j}\left(x_{i, j+1}^{l-1}+x_{i, j-1}^{l}\right)\right. \\
& \left.-\frac{1}{2} \beta_{i, j}\left(x_{i+1, j+1}^{l-1}-x_{i+1, j-1}^{l-1}-x_{i-1, j+1}^{l}+x_{i-1, j-1}^{l}\right)\right]
\end{aligned}
$$

where

$$
\begin{aligned}
\alpha_{i, j} & =\left(x_{\eta}\right)_{i, j}^{2}+\left(y_{\eta}\right)_{i, j}^{2} \\
\beta_{i, j} & =\left(x_{\xi}\right)_{i, j}\left(x_{\eta}\right)_{i, j}+\left(y_{\xi}\right)_{i, j}\left(y_{\eta}\right)_{i, j} \\
\gamma_{i, j} & =\left(x_{\xi}\right)_{i, j}^{2}+\left(y_{\xi}\right)_{i, j}^{2}
\end{aligned}
$$

and

$$
\begin{aligned}
& \left(x_{\xi}\right)_{i, j}=\left(x_{i+1, j}^{l-1}-x_{i-1, j}^{l}\right) / 2 \\
& \left(x_{\eta}\right)_{i, j}=\left(x_{i, j+1}^{l-1}-x_{i, j-1}^{l}\right) / 2 \\
& \left(y_{\xi}\right)_{i, j}=\left(y_{i+1, j}^{l-1}-y_{i-1, j}^{l}\right) / 2 \\
& \left(y_{\eta}\right)_{i, j}=\left(y_{i, j+1}^{l-1}-y_{i, j-1}^{l}\right) / 2 \\
& \text { for } i=2, \ldots, N_{1}-1 \text { and } j=2, \ldots, N_{2}-1 .
\end{aligned}
$$



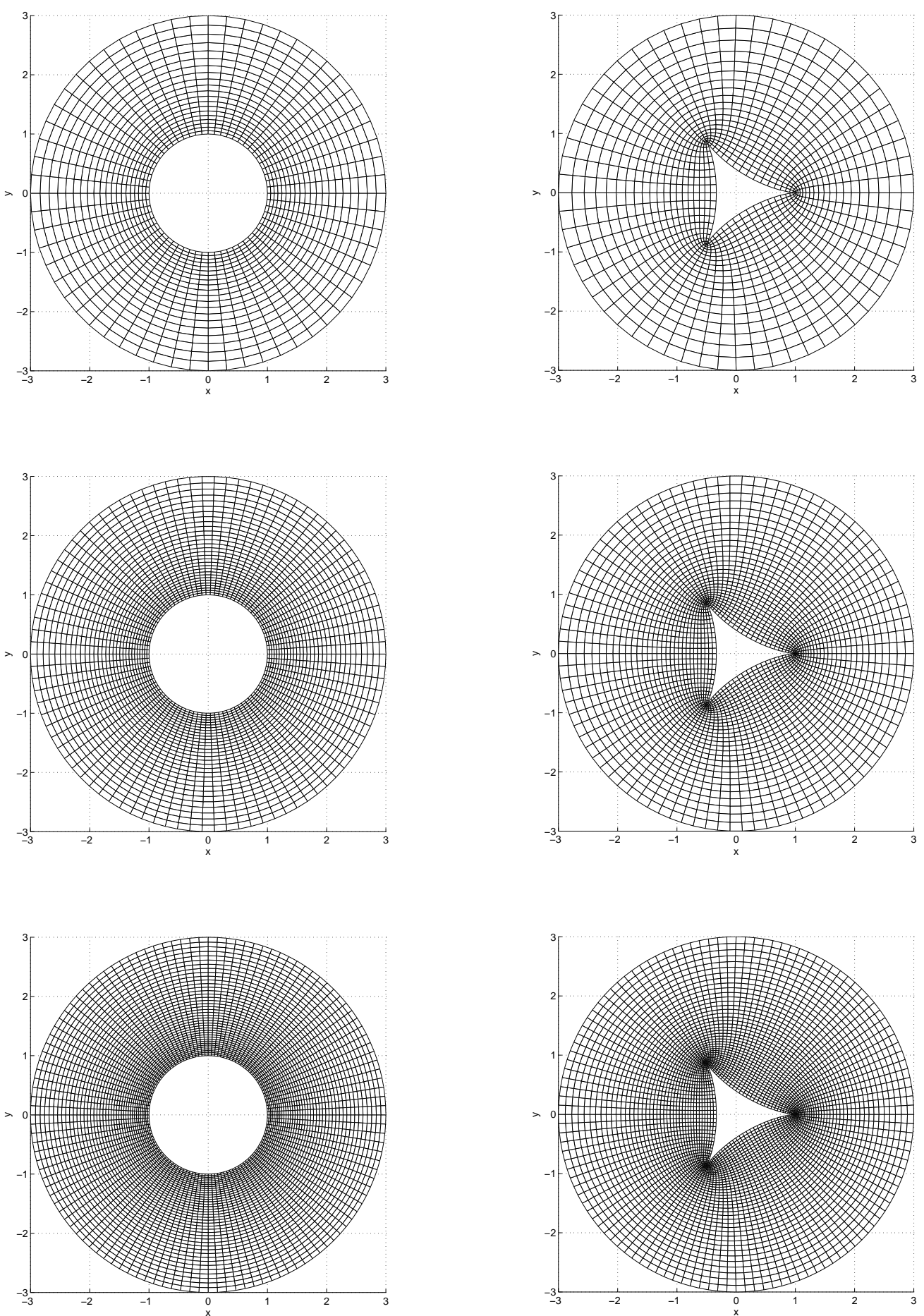

FIG. 6.1. Increasingly finer Winslow grids conforming a circle. The grid sizes (from top to bottom) are: $60 \times 20,90 \times 30$, and $120 \times 40$.
FIG. 6.2. Increasingly finer Winslow grids conforming the three-cusp Astroid. The grid sizes (from top to bottom) are: $60 \times 20,90 \times 30$, and $120 \times 40$. 
A similar algebraic equation is obtained for the discrete values of the $y$-component. The super-indices $l$ and $l-1$ represent the current and previous steps in the iteration. The SOR method uses a relaxation parameter $\varpi$, such that $x_{i, j}^{l}=\varpi x_{i, j}^{l}+(1-\varpi) x_{i, j}^{l-1}$, with an analogous equation for $y_{i, j}$. The value of $\varpi=1.65$ gives excellent convergence. Good reviews of the SOR iterative technique for finite-difference methods are found in Chapter 13 of the book by Strikwerda [20] and Chapter 4 of [16] by LeVeque. For $l=0$, an initial grid is defined. Then, new grids are iteratively obtained until the maximum pointwise error between two consecutive grids falls under some specified tolerance, ie. $\mathrm{err}^{l}<$ Tol. This maximum pointwise error is defined as follows:

$$
\operatorname{err}^{l}=\max _{\substack{1 \leq i \leq N_{1} \\ 1 \leq j \leq N_{2}}}\left\{\left|x_{i, j}^{l}-x_{i, j}^{l-1}\right|,\left|y_{i, j}^{l}-y_{i, j}^{l-1}\right|\right\} .
$$

Grids generated according to the above procedure are shown in Figures 6.1 and 6.2. These are increasingly finer grids conforming a circle and a three-cusp Astroid, respectively. The circle represents the cross-section of a model or benchmark scatterer, and the Astroid represents an obstacle with arbitrary cross-section. Its parametric curve is given by: $x(t)=(2 \cos t+\cos 2 t) / 3, y(t)=(2 \sin t-\sin 2 t) / 3$, and $0 \leq t \leq 2 \pi$. The grid sizes (from top to bottom) are: $60 \times 20,90 \times 30$, and $120 \times 40$. It is clear that any other shape could have been selected as the arbitrary scatterer.

The Winslow elliptic equations applied to these domains produce non-self-overlapping and smooth grids without the propagation of boundary slope-discontinuities into the interior. These are properties of primary importance for the numerical solution of the field variables supported by these grids. A good summary of these techniques can be found in Chapter 8 of the book by Hansen et al. [11] and the Handbook of Grid Generation by Thompson et al. [25].

For novel and more efficient alternative elliptic grid generation algorithms, the reader is referred to [27, 4, 3]. These references deal with methods that improve the quality of the grid by adding control over the cell size, improving the grid point distribution and producing clustering and/or stretching of grid lines where needed.

6.2. Numerical Scattering Problem. As discussed in the introduction, the scattering problem may be modeled by the time-dependent formulation of the telegraph Equation (1.1) or by the reduced telegraph Equation (1.2) assuming time-harmonic oscillations. In previous works [28, 3, 26], the explicit finite-difference method has been used for the time-dependent model using generalized curvilinear coordinates. In this work, we adopt the second model. The numerical method consists of an implicit finite-difference technique supported by the Winslow elliptic grids employed to perform the spatial discretization of geometrically complex regions.

A second order finite-difference discretization is adopted to approximate all the partial derivative terms in the governing Equation (5.6). As a result, a linear system of algebraic equations is obtained for the numerical approximation of the scattered field $u_{s c}$ at each grid point.

At any interior point, the algebraic equation for $\left(u_{s c}\right)_{i, j}$ has the following form:

$$
\begin{aligned}
& \left(k^{2} J_{i, j}^{2}-2\left(\alpha_{i, j}+\gamma_{i, j}\right)\right)\left(u_{s c}\right)_{i, j}+\alpha_{i, j}\left(\left(u_{s c}\right)_{i+1, j}+\left(u_{s c}\right)_{i-1, j}\right)+\gamma_{i, j}\left(\left(u_{s c}\right)_{i, j+1}+\left(u_{s c}\right)_{i, j-1}\right) \\
& -\frac{\beta_{i, j}}{2}\left(\left(u_{s c}\right)_{i+1, j+1}-\left(u_{s c}\right)_{i+1, j-1}-\left(u_{s c}\right)_{i-1, j+1}+\left(u_{s c}\right)_{i-1, j-1}\right)=0 \\
& \text { for } 1 \leq i \leq N_{1}-1 \text { and } 2 \leq j \leq N_{2} .
\end{aligned}
$$

At the artificial boundary $\mathcal{B}$, the discrete Equation (6.3) evaluated at $\left(u_{s c}\right)_{i, N_{2}}$ involves the values of the three "ghost" points denoted by $\left(u_{s c}\right)_{i-1, N_{2}+1},\left(u_{s c}\right)_{i, N_{2}+1}$ and $\left(u_{s c}\right)_{i+1, N_{2}+1}$. These ghost points also appear in the discretization of the DtN boundary condition:

$$
\begin{aligned}
& \frac{1}{R J_{i, N_{2}}}\left[\left(x_{i, N_{2}}\left(y_{\eta}\right)_{i, N_{2}}-y_{i, N_{2}}\left(x_{\eta}\right)_{i, N_{2}}\right)\left(\left(u_{s c}\right)_{i+1, N_{2}}-\left(u_{s c}\right)_{i-1, N_{2}}\right)\right. \\
& \left.+\left(y_{i, N_{2}}\left(x_{\xi}\right)_{i, N_{2}}-x_{i, N_{2}}\left(y_{\xi}\right)_{i, N_{2}}\right)\left(\left(u_{s c}\right)_{i, N_{2}+1}-\left(u_{s c}\right)_{i, N_{2}-1}\right)\right] \\
& =\frac{k \Theta^{2}}{\pi} \sum_{n=0}^{N} \sum_{i^{\prime}=1}^{N_{1}-1} \epsilon_{n} \frac{H_{n}^{(1)}{ }^{\prime}(k R)}{H_{n}^{(1)}(k R)} \cos n \Theta\left(i-i^{\prime}\right)\left(u_{s c}\right)_{i^{\prime}, N_{2}} \\
& \text { for } 1 \leq i \leq N_{1}-1,
\end{aligned}
$$


where the symbol $\Theta=2 \pi /\left(N_{1}-1\right)$. This relation is valid for an equidistant distribution of grid points along $\mathcal{B}$ where the integral operator has been approximated by the trapezoidal quadrature rule. This is proven to have second order convergence for periodic functions. The factor $\Theta^{2}$ arose from the trapezoidal rule and from the fact that $\theta^{\prime}(\bar{\xi})=\Theta$ for this particular parametrization of the circle $\mathcal{B}$. Also, notice that the DtN series has been truncated to include only up to $N$ terms.

At points on the obstacle's boundary, the following discrete equations are derived. For the soft cylinder:

$$
\begin{aligned}
& \left(u_{s c}\right)_{i, 1}=\frac{\sqrt{-1} \Lambda}{4} H_{0}^{(1)}\left(k \tilde{r}_{i}\right) \\
& \text { for } 1 \leq i \leq N_{1}-1,
\end{aligned}
$$

and for the hard cylinder:

$$
\gamma_{i, 1}\left(-3\left(u_{s c}\right)_{i, 1}+4\left(u_{s c}\right)_{i, 2}-\left(u_{s c}\right)_{i, 3}\right)-\beta_{i, 1}\left(\left(u_{s c}\right)_{i+1,1}-\left(u_{s c}\right)_{i-1,1}\right)=\frac{\sqrt{-1} \Lambda k \mu_{i} J_{i, 1}}{2 \tilde{r}_{i}} H_{0}^{(1) \prime}\left(k \tilde{r}_{i}\right)(6.6)
$$

for $1 \leq i \leq N_{1}-1$

where the term $\left(u_{s c}\right)_{\eta}$ from Equation (5.10) was discretized using a forward second order finite-difference formula. This was done in order to preserve the second order scheme and avoid the introduction of ghost points at the obstacle's boundary. The symbols $\tilde{r}_{i}$ and $\mu_{i}$ appearing in Equations (6.5)-(6.6) are defined as follows:

$$
\begin{array}{r}
\tilde{r}_{i}=\sqrt{\left(x_{i, 1}-x_{s}\right)^{2}+\left(y_{i, 1}-y_{s}\right)^{2}} \\
\mu_{i}=\left(y_{i, 1}-y_{s}\right)\left(x_{\xi}\right)_{i, 1}-\left(x_{i, 1}-x_{s}\right)\left(y_{\xi}\right)_{i, 1} .
\end{array}
$$

The algebraic system formed by all these equations is then solved using a MATLAB direct solver subroutine for linear systems. The number of unknowns is equal to the number of grid points (without counting twice the points at the branch cut) plus the number of ghost points. In terms of the grid size, there are $\left(N_{1}-1\right) \times\left(N_{2}+1\right)$ equations and unknowns. The matrix pattern obtained from this finite-difference discretization, including the obstacle's boundary and ghost points, is shown in Figure 6.3. The algebraic system of equations (and the associated matrix) is formed in the following manner. Equations (6.5) or (6.6) are used starting from the first node $(i=1)$ at the obstacle's boundary curve $(j=1)$ until the last node of that $j$-level where $i=N_{1}-1$. Then, Equation (6.3) is used starting at $i=1$ and $j=2$, moving outward in increasing order of $i$ and $j$ until we reach the last node of the artificial boundary $\mathcal{B}$ where $i=N_{1}-1$ and $j=N_{2}$. At this point, the discrete DtN Equation (6.4) is employed to complete the algebraic system and to determine the $N_{1}-1$ ghost points. Since the DtN condition is global in character, a full block is obtained at the lower-right corner of the matrix.

7. Far-Field Pattern. In many applications, it is important to describe the wave field far from the sources and scatterers. For that reason, a function known as the far-field pattern is commonly used to characterize the scattered field. The solution $u_{s c}$ to the BVP (3.1)-(3.4) has the following asymptotic behavior:

$$
u_{s c}(r, \theta) \rightarrow \frac{e^{i k r}}{\sqrt{k r}} f(\theta, k) \quad \text { as } \quad r \rightarrow \infty,
$$

where $f(\theta, k)$ defines the far-field pattern. The magnitude of $f(\theta, k)$ represents the relative amplitude of the scattered field as a function of the angle $\theta$ and the effective wave number $k$. Scatterers with different shape produce different far-field patterns in general.

In previous sections, analytical and numerical solutions to the scattering problem have been obtained. In both cases, the scattered field has been represented in terms of Hankel functions expansions in the exterior sub-domain $\Omega_{\text {far }}$. Therefore, by taking advantage of the asymptotic behavior of the Hankel functions, the far-field pattern can be calculated. Indeed, Equation (3.11) states that

$$
H_{n}^{(1)}(k r) \rightarrow \frac{e^{i k r}}{\sqrt{k r}}(-i)^{n} \frac{(1-i)}{\sqrt{\pi}}, \quad \text { as } \quad r \rightarrow \infty
$$



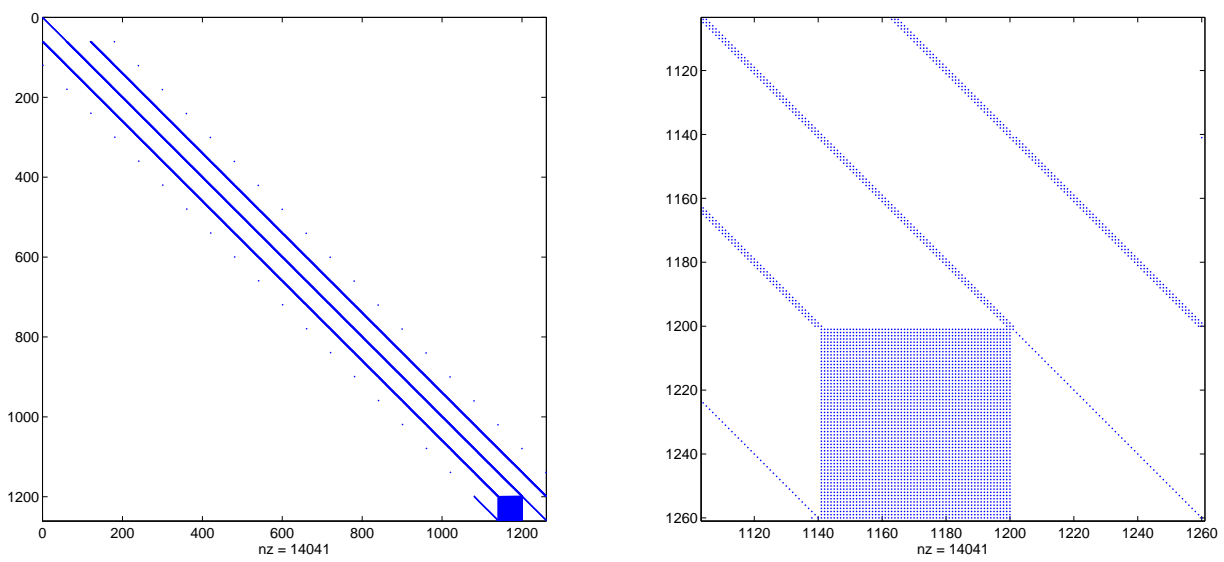

FIG. 6.3. Finite-difference matrix pattern for the scattering problem yielded on a Winslow grid. The full matrix is shown on the left and a zoom on the lower-right corner is shown on the right. The bottom block of this matrix corresponds to the discrete equations derived from the DtN boundary condition. These involve the values at the "ghost" points and all grid points on the artificial boundary.

For the circular cylindrical case, the far-field pattern can be obtained by substituting the above expansion (7.2) into the analytical solution (3.10). This yields

$$
f(\theta, k)=\frac{\Lambda(1+i)}{4 \sqrt{\pi}} \sum_{n=0}^{\infty} \epsilon_{n} i^{n} C_{n} H_{n}^{(1)}(k L) \cos n(\theta-\phi) .
$$

On the other hand, for the numerical treatment, substitution of the asymptotic expansions of the Hankel functions (7.2) into the semi-analytical solution (4.1) in $\Omega_{\text {far }}$ leads to

$$
f(\theta, k)=\frac{(1-i)}{2 \pi \sqrt{\pi}} \sum_{n=0}^{\infty} \frac{\epsilon_{n}(-i)^{n}}{H_{n}^{(1)}(k R)} \int_{0}^{2 \pi} u_{s c}(R, \bar{\theta}) \cos n(\theta-\bar{\theta}) d \bar{\theta} .
$$

Approximations can be obtained from (7.3) and (7.4) by employing a finite number of terms of the infinite series and by using the trapezoidal rule for the integrals. We stress the importance that the far-field pattern is a function of $k$ as much as it is of $\theta$. The effective wave number $k$ has embedded the values of the damping and stiffness coefficients. So, it is the far-field pattern what is analyzed in Section 8 to show the influence of energy dissipation and media stiffness on the wave fields.

8. Validation and Results. In this section, the numerical method is first checked by comparing its approximate solution with the exact solution for a benchmark problem. More specifically, comparisons are made for the far-field pattern. After that, series of far-field patterns and near-field solutions, resulting from several values of damping and stiffness coefficients, are presented.

8.1. Convergence of the Numerical Method. The benchmark problem for this work is the scattering from a circular cylinder of radius $a=1$, and angular frequency $w=2 \pi$. In the global Cartesian coordinates, the cylinder is centered at the origin, and the source of strength $\Lambda=50$ is located at $\left(x_{s}, y_{s}\right)=(-6,0)$. This configuration is equivalent to set $\phi=0$ and $L=6$. For the numerical implementation, $R=2, N=40$, and $T o l=1 e-6$ are chosen. The damping and stiffness coefficients are selected to represent all three wave regimes or cases. For Case 1: $A=0$ and $B=40$, for Case 2: $A=0$ and $B=5$, and for Case $3: A=1$ and $B=5$.

The error of the numerical solution is measured in terms of the maximum pointwise difference between the far-field pattern obtained analytically and numerically from Equations (7.3) and (7.4), respectively. To analyze the convergence of the numerical method, the grids are refined by increasing $N_{1}$ while keeping $N_{1}=4 N_{2}$. The error versus $N_{1}$ is shown in Figure 8.1. These results corroborate the second order convergence expected from the finitedifference scheme. 

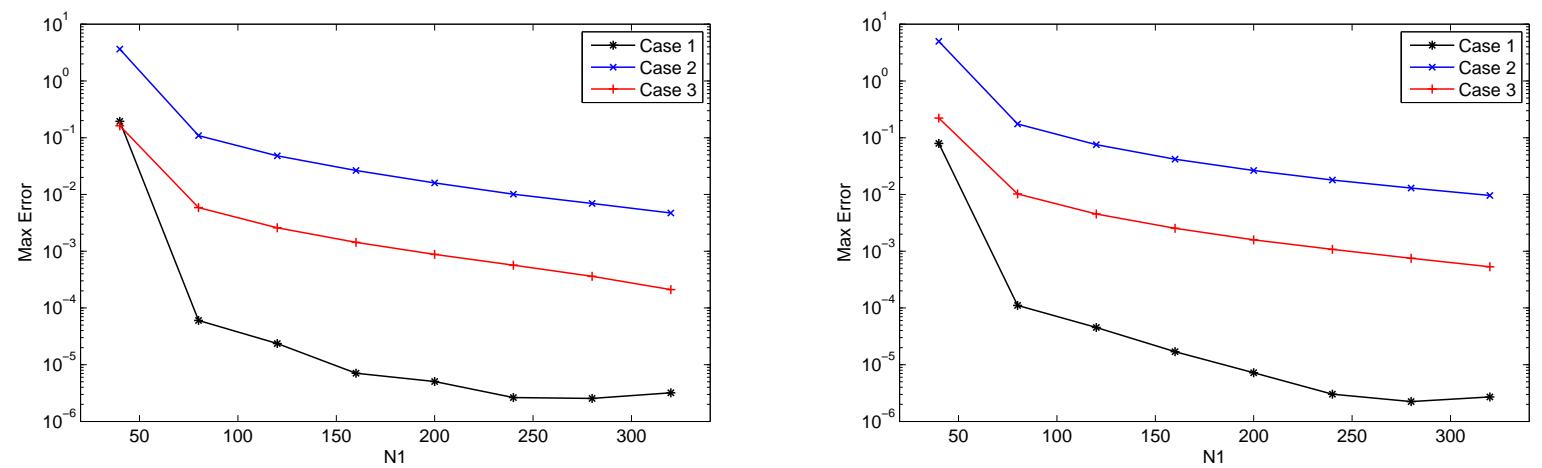

FIG. 8.1. The maximum absolute error for the numerically computed far-field pattern vs. the number of grid points $N_{1}=4 N_{2}$. Scattering from a circular cylinder of radius $a=1$ and frequency $\omega=2 \pi$. Results for both soft (left) and hard (right) cylinders. The damping and stiffness coefficients are selected to represent all three wave regimes or cases. For Case 1: $A=0$ and $B=40$, for Case 2: $A=0$ and $B=5$, and for Case 3: $A=1$ and $B=5$.

8.2. Energy Dissipation and Media Stiffness. First, the solutions for the wave scattering from a circular cylinder for various values of the damping and stiffness coefficients are displayed. These results are obtained from the analytical expression (7.3) of the far-field pattern. Figures 9.1 and 9.2 (soft and hard cylinders, respectively) show how the far-field changes as the damping coefficient $A$ increases, while keeping $B=0$. As expected, the amplitude of the pattern decreases in all directions due to energy dissipation. However, its shape remains approximately the same. On the other hand, Figures 9.3 and 9.4 show the variations of the far-field pattern as the stiffness coefficient $B$ increases, while keeping $A=0$. In this case, the shape of the pattern is transformed as its dependence on the angle $\theta$ changes. The amplitude becomes larger in some directions and smaller in others.

Secondly, variations of the far-field pattern with respect to damping and stiffness coefficients are also obtained for a three-cusp Astroid. The results are obtained from Equation (7.4). The calculations are performed with the following fixed parameters: $c=1, \omega=2 \pi, \phi=0, L=6, R=2, N=40, T o l=1 e-6$, and grid size $180 \times 30$. The Figures 9.5 and 9.6 show the influence of the damping coefficient for the hard and soft cylinders, respectively. Also, Figures 9.7 and 9.8 display the amplitude variations due to stiffness. Again, energy dissipation does not change the shape of the far-field pattern but only its amplitude, while media stiffness affects both, the amplitude and the pattern dependence on the angular direction $\theta$.

The amplitude of the wave field in the vicinity of the scatterer is also an important element of the solution. For that reason, contour representations of the amplitude of the total field have been included. These are displayed in Figure 9.9 for increasing values of damping and in Figure 9.10 for increasing values of stiffness. In both cases, the cylinder with soft surface is considered. The counterparts for the hard surface are shown in Figures 9.11 and 9.12, respectively. For these experiments, a larger computational domain with $R=3$ (grid size: $180 \times 60$ ) has been chosen in order to visualize the wave fields in an extended region around the scatterer.

The contour representations of the solution include a color scale of the wave amplitude. This helps to distinguish how the field amplitude is attenuated as the damping coefficient increases, and how the wavelength becomes larger as the stiffness coefficient is incremented. In the limiting case, when $B$ becomes too large, the spatial oscillatory profile is lost as it was described in Section 3.

One of the main features of the proposed numerical scheme is its computational feasibility. The major computational effort consists of two numerical processes. First, the grid generation process based on the point-SOR iterative method. Second, the numerical solution of the matrix equation to obtain the discrete values of $u_{s c}$. MATLAB uses a direct method based on LU-decomposition by Gaussian elimination with pivoting. This algorithm has worked accurately for the medium size matrices involved in the numerical scattering problem. The number of SOR iterations and the CPU execution time are displayed in Table 8.1 for the scattering from the three-cusp Astroid cylinder supported by increasingly finer curvilinear grids. Our code was written in MATLAB R2007a environment and executed on a Mac PowerPC G5 (1.1) with CPU speed of $2.5 \mathrm{GHz}$. 
TABLE 8.1

Numerical results for the scattering from a three-cusp Astroid obstacle. Grids are generated with $T o l=1 e-6$ and $R=2$.

\begin{tabular}{cccc}
\hline Grid-size & SOR-iter & $\begin{array}{c}\text { Grid generation } \\
\text { CPU time }(\mathrm{sec})\end{array}$ & $\begin{array}{c}\text { Linear system } \\
\text { CPU time }(\mathrm{sec})\end{array}$ \\
\hline $60 \times 10$ & 108 & 0.14 & 0.03 \\
$120 \times 20$ & 172 & 0.73 & 0.15 \\
$180 \times 30$ & 350 & 2.98 & 0.42 \\
$240 \times 40$ & 577 & 8.68 & 0.95 \\
\hline
\end{tabular}

9. Conclusion. We have studied the time-harmonic wave scattering from cylindrical obstacles. To account for damping and media stiffness, the governing relation of the mathematical model is the telegraph equation. A numerical method was developed for arbitrarily shaped cylindrical obstacles. The most important features of the proposed numerical scheme may be summarized as follows:

(i) It has second order convergence to the exact solution for the circular cylindrical obstacle (soft and hard). Moreover, it reproduces the physical behavior observed from the exact solution for all the three cases described in Section 3. The numerical far-field pattern shows the same dependence on the damping $A$ and the stiffness $B$ coefficients, as the far-field obtained from the analytical solution does.

(ii) Scattering from truly arbitrary obstacles can be numerically modeled due to the re-formulation of the problem in generalized curvilinear coordinates supported on Winslow elliptic grids. These meshes conform to the geometry of the obstacle. There is no need for staircase approximations which introduce additional error.

(iii) The exact DtN non-reflecting boundary condition was also reformulated in terms of generalized curvilinear coordinates and used as an exterior boundary condition for the BVP to be solved numerically. As a consequence, the computational domain is greatly reduced while the numerical method maintains its accuracy. Additionally, the far-field pattern is easily obtained from the algebraic expressions of the DtN condition.

This work is being extended to the treatment of several scatterers supported by elliptic grids of higher quality. This extension shall be presented in a forthcoming paper.

Acknowledgments. The authors would like to acknowledge and thank Dr. Vianey Villamizar, of the Brigham Young University Department of Mathematics, for his indispensable guidance and support. They also thank the referees of this paper for their most helpful suggestions. 


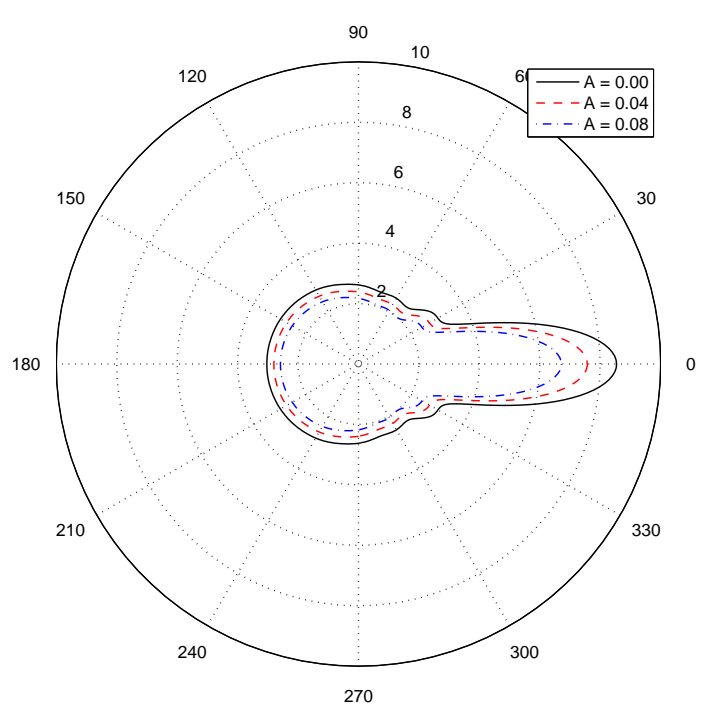

FIG. 9.1. Far-field patterns corresponding to the soft circular cylinder for various values of damping coefficient $A$, while keeping $B=0$.

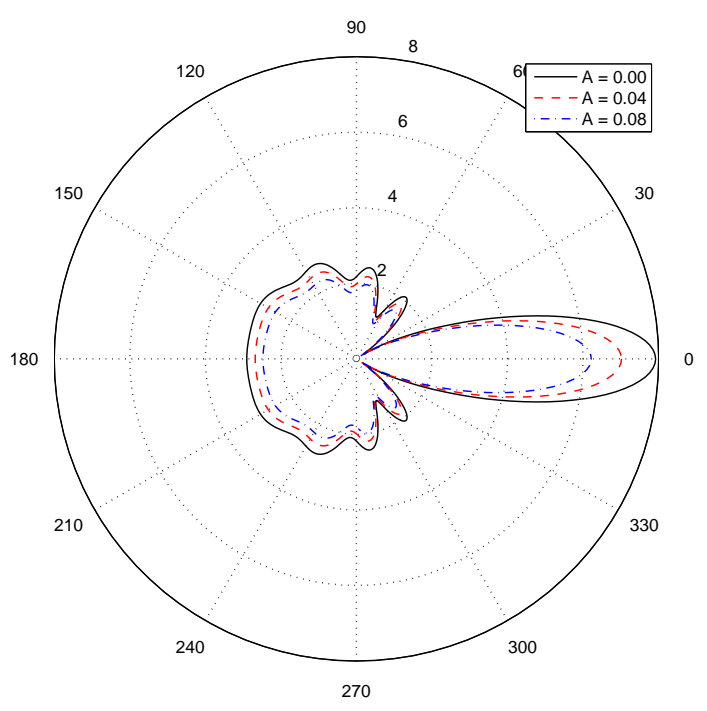

FIG. 9.2. Far-field patterns corresponding to the hard circular cylinder for various values of damping coefficient $A$, while keeping $B=0$.

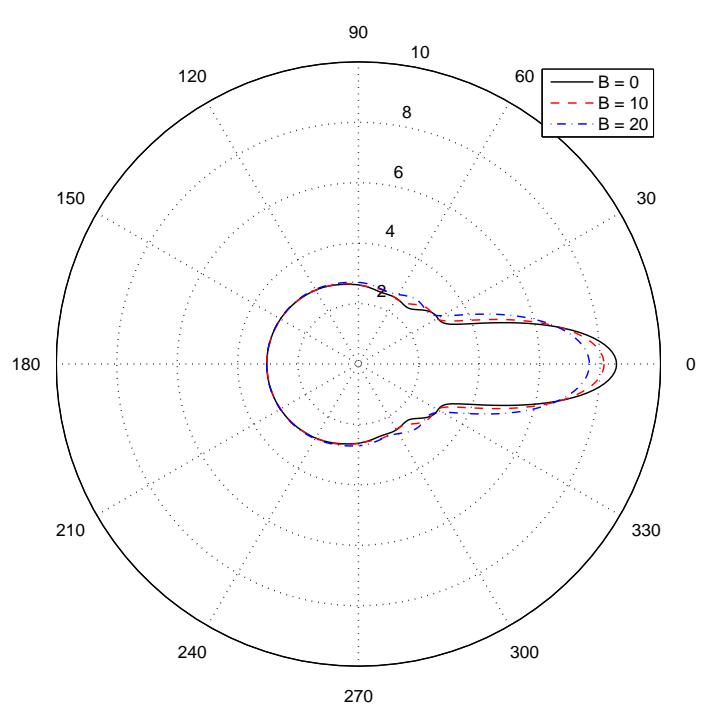

FIG. 9.3. Far-field patterns corresponding to the soft circular cylinder for various values of stiffness coefficient $B$, while keeping $A=0$.

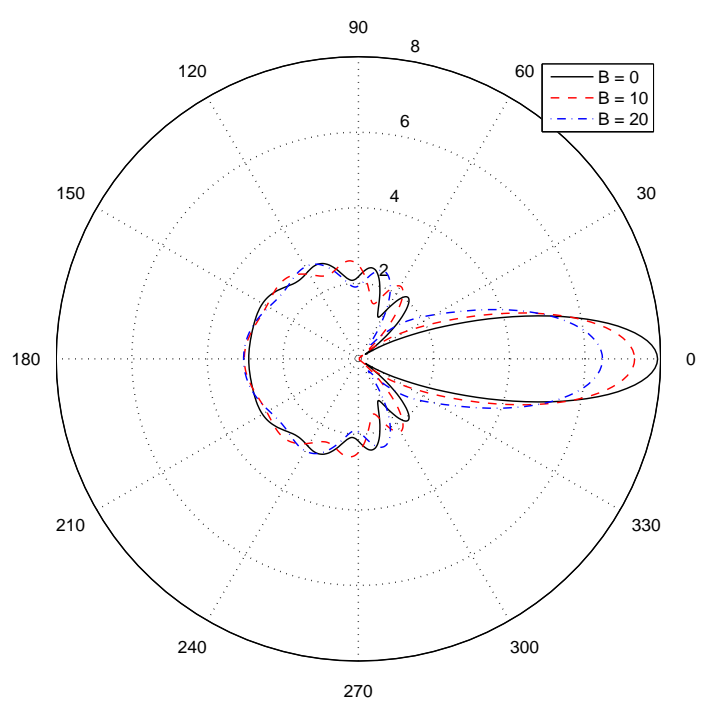

FIG. 9.4. Far-field patterns corresponding to the hard circular cylinder for various values of stiffness coefficient $B$, while keeping $A=0$. 


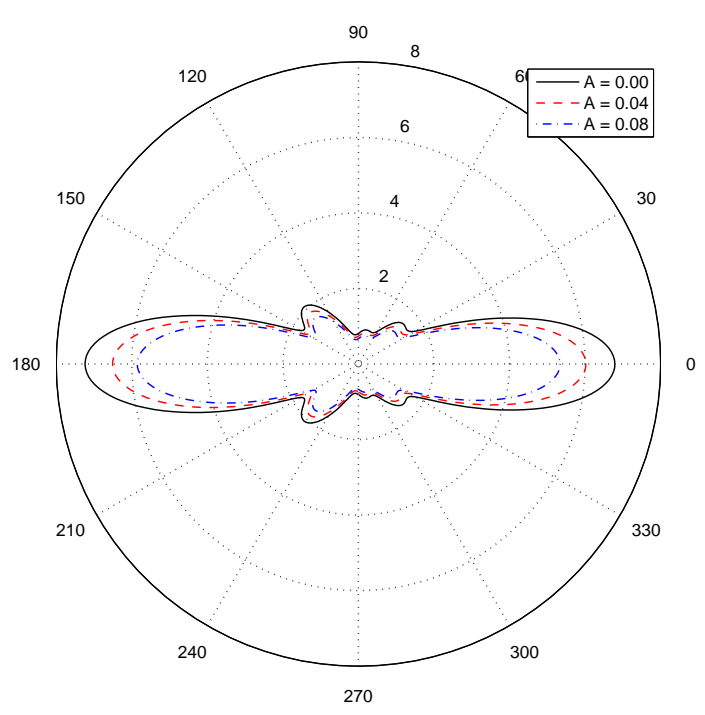

FIG. 9.5. Far-field patterns corresponding to the soft three-cusp Astroid cylinder for various values of damping coefficient $A$, while keeping $B=0$.

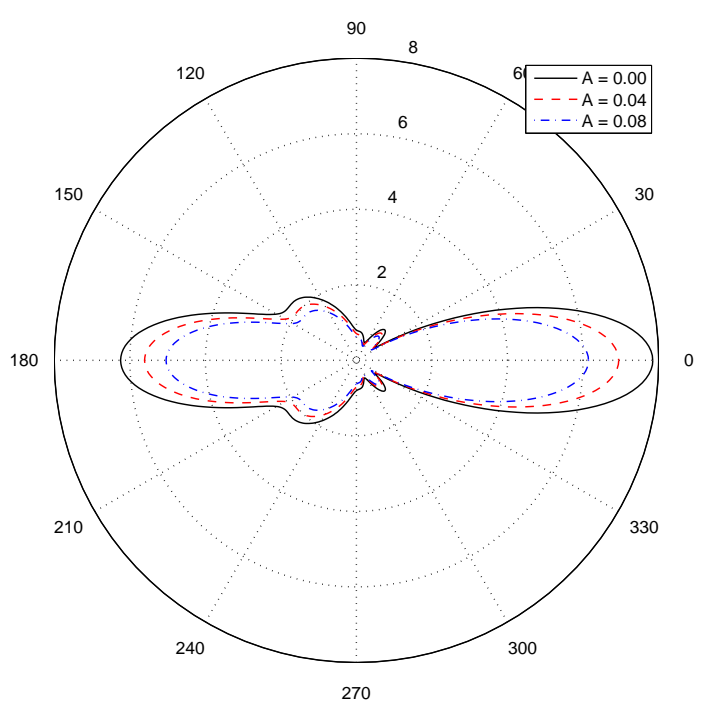

FIG. 9.6. Far-field patterns corresponding to the hard three-cusp Astroid cylinder for various values of damping coefficient $A$, while keeping $B=0$.

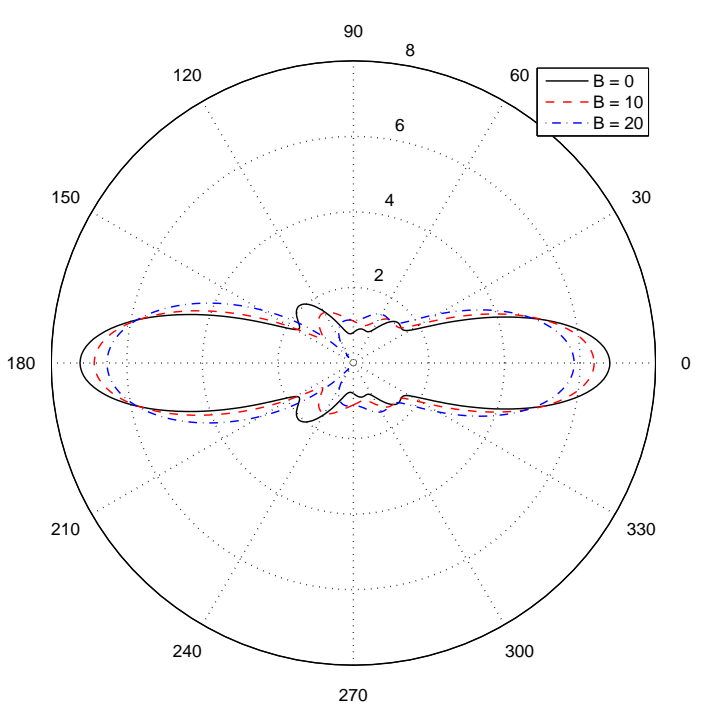

FIG. 9.7. Far-field patterns corresponding to the soft three-cusp Astroid cylinder for various values of stiffness coefficient $B$, while keeping $A=0$.

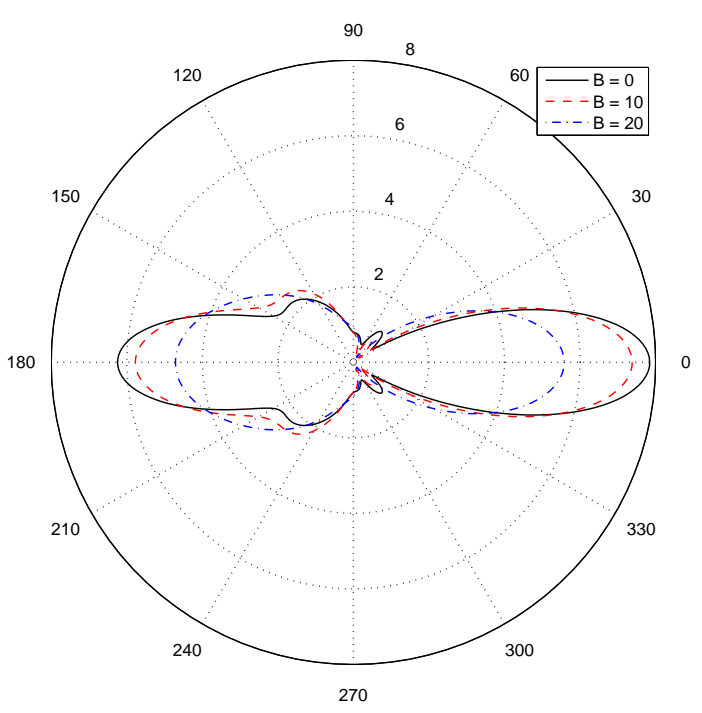

FIG. 9.8. Far-field patterns corresponding to the hard threecusp Astroid cylinder for various values of stiffness coefficient $B$, while keeping $A=0$. 

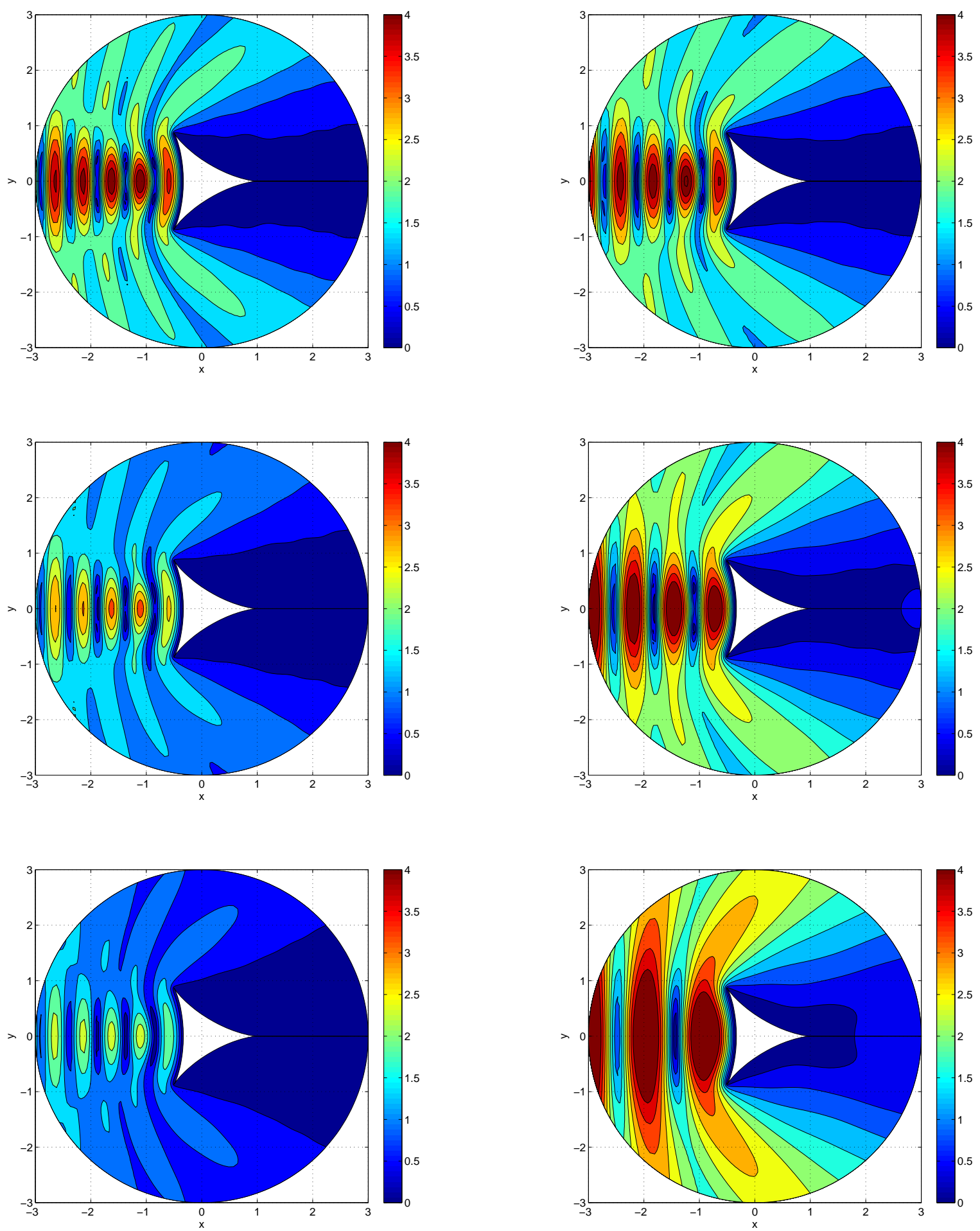

FIG. 9.9. Amplitude of the total wave field in the vicinity of the soft three-cusp Astroid cylinder. From top to bottom $A=$ $0.0,0.1,0.2$, while keeping $B=0$.
FIG. 9.10. Amplitude of total wave field in the vicinity of the soft three-cusp Astroid cylinder. From top to bottom $B=10,20,30$, while keeping $A=0$. 

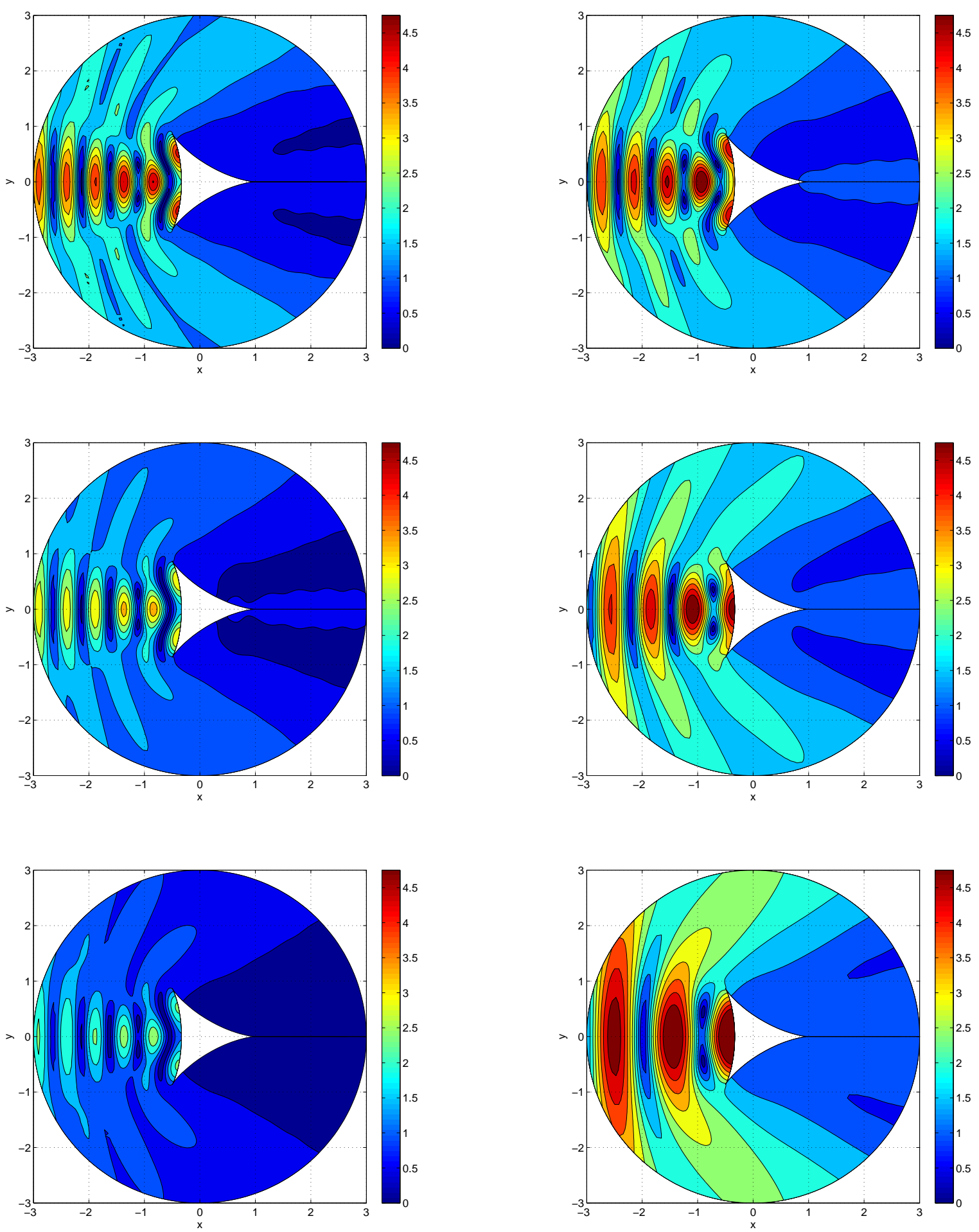

FIG. 9.11. Amplitude of the total wave field in the vicinity of the hard three-cusp Astroid cylinder. From top to bottom $A=0.0,0.1,0.2$, while keeping $B=0$.
FIG. 9.12. Amplitude of the total wave field in the vicinity of the hard three-cusp Astroid cylinder. From top to bottom $B=10,20,30$, while keeping $A=0$. 


\section{REFERENCES}

[1] M.A. Abdou. A domian decomposition method for solving the telegraph equation in charged particle transport. Journal of Quantitative Spectroscopy and Radiative Transfer, 95:407-414, 2005.

[2] M. Abramowitz and I. Stegun, editors. Handbook of Mathematical Functions with Formulas, Graphs, and Mathematical Tables. Dover Publications, 9th edition, 1970.

[3] S. Acosta and V. Villamizar. Acoustic scattering approximations on elliptic grids with adaptive control functions. In Proc. 8th Int. Conf. on Math. and Num. Aspects of Waves, pages 514-516, Reading, UK, 2007.

[4] S. Acosta and V. Villamizar. Grid generation with grid line control for regions with multiple complexly shaped holes. In Proceedings MASCOTO6 IMACS Series in Comp. and Appl. Math., volume 11, pages 1-12, Rome, Italy, 2007.

[5] A. Barletta and E Zanchini. A thermal potential formulation of hyperbolic heat conduction. ASME J. Heat Transfer, 121:166-169, 1999.

[6] K.J. Baumeister and T.D. Hamill. Hyperbolic heat conduction equation - a solution for the semi-infinite body problem. ASME J. Heat Transfer, 91:543-548, 1969.

[7] C.I. Christov. On the evolution of localized wave packets governed by a dissipative wave equation. Wave Motion, 45:154-161, 2008.

[8] Y. Fedorov and B.A. Shakhov. Description of non-diffusive solar cosmic ray propagation in a homogeneous regular magnetic field. Astronomy and Astrophysics, 402:805-817, 2003.

[9] L. Flax and W. Neubauer. Reflection of elastic waves by a cylindrical cavity in an absorptive medium. J. Acoust. Soc. Am., 63(3):675-680, 1978.

[10] T.I. Gombosi, J.R. Jokipii, J. Kota, K. Lorencz, and L.L. Williams. The telegraph equation in charged particle transport. The Astrophysical Journal, 403:377-384, 1993.

[11] G. A. Hansen, R. W. Doglass, and A. Zardecki. Mesh Enhancement. Imperial College Press, 2005.

[12] E. Holmes. Are diffusion models too simple? a comparison with telegraph models of invation. The American Naturalist, 142(5):779-795, 1993.

[13] M.N. Ichchou, A. Le Bot, and Jezequel L. A transient local energy approach as an alternative to transient sea: Wave and telegraph equations. Journal of Sound and Vibration, 246(5):826-840, 2001.

[14] J. Keller and D. Givoli. Exact non-reflecting boundary conditions. J. Comput. Phys., 82:172-192, 1989.

[15] P. Knupp and S. Steinberg. Fundamentals of Grid Generation. CRC Press, 1993.

[16] R.J. LeVeque. Finite Difference Methods for Ordinary and Partial Differential Equations. SIAM, 1st edition, 2007.

[17] P. Martin. Multiple Scattering : Interaction of Time-Harmonic Waves with N Obstacles. Cambridge University Press, 2006.

[18] G.F. Roach. Green's Functions. Cambridge University Press, 2nd edition, 1995.

[19] E. Rodarte, G. Singh, Miller N.R., and P. Hrnjak. Sound attenuation in tubes due to visco-thermal effects. Journal of Sound and Vibration, 231(5):1221-1242, 2000.

[20] J.C. Strikwerda. Finite Difference Schemes and Partial Differential Equations. SIAM, 2nd edition, 2004.

[21] N. Sushilov and R. Cobbold. Wave propagation in media whose attenuation is proportional to frequency. Wave Motion, 38:207-219, 2003.

[22] T. Szabo. Time domain wave equations for lossy media obeying a frequency power law. J. Acoust. Soc. Am., 96(1):491-500, 1994.

[23] T. Szabo and J. Wu. A model for longitudinal and shear wave propagation in viscoelastic media. J. Acoust. Soc. Am., 107(5):2439-2446, 2000.

[24] S. Temkin. Viscous attenuation of sound in dilute suspensions of particles. J. Acoust. Soc. Am., 100(2):825-831, 1996.

[25] J. F. Thompson, B. K. Soni, and N. G. Weatherill. Handbook of Grid Generation. CRC Press, 1999.

[26] V. Villamizar and S. Acosta. Generation of smooth grids with line control for scattering from multiple obstacles. Math. Comput. Simul., 2008. In press.

[27] V. Villamizar, O. Rojas, and J. Mabey. Generation of curvilinear coordinates on multiply connected regions with boundary singularities. $J$. Comput. Phys., 223:571-588, 2007.

[28] V. Villamizar and M. Weber. Boundary-conforming coordinates with grid line control for acoustic scattering from complexly shaped obstacles. Numer. Meth. Part. Differ. Equ., 23:1445-1467, 2007.

[29] G.N. Watson. A Treatise on the Theory of Bessel Functions. Cambridge University Press, 2nd edition, 1962.

[30] A. Winslow. Numerical solution of the quasilinear poisson equations in a nonuniform triangle mesh. J. Comp. Phys., 2:149-172, 1967. 